\title{
The diagnostic value of PET/CT imaging with the 68Ga-labeled PSMA-ligand in the follow up assessment of prostate cancer after therapy
}

\author{
Talaat Ahmed Abd El Hameed Hassan ${ }^{1 *}$ (i) and Mohammed Samy Elazab ${ }^{2}$
}

\begin{abstract}
Background: The aim of this study is to investigate the role of PET/CT imaging with the 68Ga-labeled PSMA-ligand in the follow up assessment of post-operative and post-therapeutic cases of prostate cancer. All data were collected and analyzed retrospectively, enrolling 30 patients (their age range from 53 to 80 years with a mean age 67.8). The patients had been referred to a private imaging center for their assessment by 68Ga-labeled PSMA-ligand PET/CT.

Results: By 68Ga-labeled PSMA-ligand PET/CT, prostatic recurrent/residual neoplastic lesion was depicted in 23 (76.6\%) patients, nodal metastases was present in 7 (23.3\%) patients, and distant metastasis was present in 11 (36.6\%) patients. The clinical and/or PSA level and/or radiological follow-up and/or the histopathological assessment for the patients served as a reference in the present study.The 68Ga-labeled PSMA-ligand PET/CT had sensitivity, specificity, positive predictive value, negative predictive value, and an overall accuracy $(96.5 \%, 100 \%, 100 \%, 50 \%$, and $96.6 \%$ ) respectively.

Conclusion: 68Ga-labeled PSMA-ligand PET/CT is a very helpful tool for detection of prostatic cancer residual/ recurrent lesions and restaging which can help in performing treatment plans and improve the clinical outcome and survival of the patients.
\end{abstract}

Keywords: Prostatic cancer, PSMA, PET/CT, 68-Ga

\section{Background}

Prostate cancer is the most common neoplasm in men worldwide and an increasing number of cases has been noted in recent years [1]. Tumor recurrence/residual after prostatectomy, radiotherapy, or other local treatment modalities represents a great obstacle in the management of prostatic cancer. Usually, post-therapeutic recurrence, after prostatectomy or external beam radiation therapy, can be diagnosed by prostate-specific antigen (PSA) values of $\geq 0.2 \mathrm{ng} / \mathrm{mL}$ in two succesive tests and this is called biochemical recurrence [2].

\footnotetext{
* Correspondence: talaathassan38@yahoo.com

${ }^{1}$ Radiology Department, Faculty of Medicine, Cairo University, Cairo, Egypt Full list of author information is available at the end of the article
}

Early diagnosis of recurrent prostatic neoplastic lesions gives better chance for early treatment and better prognosis. Also, if the recurrent tumor is localized, it can be managed by surgery or external radiation therapy and systemic therapy and its side effects can be avoided or delayed. So, an accurate diagnostic modality with high sensitivity and specificity is needed to be able to do this approach [3].

Morphologic imaging methods, like transrectal ultrasound or CT, show limitations and their sensitivity ranges between 25 and $54 \%$ for the detection of local recurrence, and these parameters are moderately improved using functional MR imaging techniques [4,5].Also, the CT or MR shows sensitivity about $30-80 \%$ for diagnosis of lymph node metastases [6].

\section{Springer Open}

(๑) The Author(s). 2020 Open Access This article is licensed under a Creative Commons Attribution 4.0 International License, which permits use, sharing, adaptation, distribution and reproduction in any medium or format, as long as you give appropriate credit to the original author(s) and the source, provide a link to the Creative Commons licence, and indicate if changes were made. The images or other third party material in this article are included in the article's Creative Commons licence, unless indicated otherwise in a credit line to the material. If material is not included in the article's Creative Commons licence and your intended use is not permitted by statutory regulation or exceeds the permitted use, you will need to obtain permission directly from the copyright holder. To view a copy of this licence, visit http://creativecommons.org/licenses/by/4.0/. 
Most prostate cancers use non-glucose metabolic pathways such as fatty acid metabolism or fructose metabolism.So FDG, which is the most widely used PET tracer in oncologic imaging, is of limited use in prostate cancer $[7,8]$.

Various PET tracers have been used to improve the detection of recurrent prostate cancer and mainly $11 \mathrm{C}$ and 11F-labeled choline tracers have been used in the past [7-9]. However, the detection rate of these tracers is only $40-60 \%$, especially in patients with PSA values below $3 \mathrm{ng} / \mathrm{mL}[5,9,10]$.

Therefore, PET/CT imaging with the 68Ga-labeled PSMA-ligand, as new and improved imaging method for prostate cancer, has recently received increased attention [11-15]. PSMA is a cell surface protein that is significantly overexpressed in prostatic cells. Other tissues, like salivary gland, proximal small intestine, and kidney, express PSMA but to a lesser extent compared to prostatic cells $[16,17]$. It therefore provides a promising target for prostate cancer specific imaging and therapy [18-25].

\section{Methods}

All data were collected and analyzed in a retrospective pattern; enrolling 30 patients who had 68Ga-labeled PSMA-ligand PET-CT examination in the period from September 2018 till February 2020; they were cases of treated prostatic malignancy. All patients were referred to a private imaging center for their assessment by 68Ga-labeled PSMA-ligand PET/CT and for the evaluation of their treatment response. Patients gave informed consent prior to the PET/CT scan. The study was approved by the local research ethical committee.

\section{Inclusion criteria}

Cases of prostatic cancer who had curative surgical resection (prostatectomy), chemotherapy, radiotherapy, hormonal therapy, or any combination of them.

\section{Exclusion criteria}

The patients who had prostatic malignancy but had not received any treatment were excluded.

\section{Physical and biological background of PSMA}

PSMA is a large transmembrane glycoprotein with a small intracellular and transmembrane component and a large extracellular component. PSMA expression is 1001000 times more in prostate cancer than other tissues, even benign prostate cells, and PSMA expression increases with increasing tumor stage and grade and in castration-resistant prostate cancers. The name PSMA is conflicting, because PSMA expression is not specific to prostate cells or prostate cancer. PSMA expression has been reported in unrelated benign and malignant conditions in a number of case reports [26].
68Ga-labeled PSMA-ligand targets the extracellular domain of PSMA with strong binding affinity and is internalized into the endosomal recycling system, with rapid blood clearance and low background activity, characteristics that lead to high-quality images with high tumor-to-background ratios. Yet, $5 \%$ of prostatic cancers do not show significant 68Ga-labeled PSMA-ligand uptake [27].

Physiologic uptake of 68Ga-labeled PSMA-ligand is seen in:

(a) The lacrimal and salivary glands, liver, spleen, kidneys, and intestine [21].

(b) Celiac ganglia, which are located in front of the crura in a para-aortic location and should not be misinterpreted as metastatic lymph nodes [28].

(c) Cervicothoracic ganglion, which lies anterior to the transverse process of the seventh cervical vertebra, inferior to the subclavian artery and superior to the neck of the first rib [29].

(d) Bone marrow 68Ga-PSMA activity is less than 18FFDG activity, a finding that potentially allows better detection of bone metastases with 68Ga-PSMA ligands [27].

(e) Unbound 68Ga-PSMA tracer is excreted by the kidneys into the ureters and urinary bladder [27].

- Focal physiologic ureteric activity can limit the assessment of small adjacent pelvic or retroperitoneal nodes [27].

- Physiologic urinary bladder activity may mask the prostate bed, particularly if the SUV threshold has not been adequately adjusted [27].

- Physiologic urethral activity of 68GaPSMA-especially after treatment with highintensity focused ultrasound or transurethral resection of the prostate-can mask or mimic disease recurrence [27].

\section{Imaging technique}

- No preparation is required before 68Ga-labeled PSMA-legand injection.

- 68Ga has a half-life of $68 \mathrm{~min}$.

Table 1 Demonstrating the range of age in years

\begin{tabular}{ll}
\hline Age & 67.8 \\
\hline Mean & 53 \\
\hline Minimum & 80 \\
\hline
\end{tabular}


- A dose of $2 \mathrm{MBq} / \mathrm{kg}$ of $68 \mathrm{Ga}-\mathrm{PSMA}$ was injected intravenously and then the patients were relaxed for $1 \mathrm{~h}$ that was considered as the uptake period.

- The PET/CT system using a multi-detector (sixteen detectors) CT machine (GE, Discovery IQ, USA) was used for the patients' examination.

- For the sake of attenuation correction and image fusion, non-contrast enhanced CT images were taken.

- Then PET scan was done and the examination levels were extending from the vertex to the knee levels.

- The total acquisition time for the integrated PET/ CT scan was 20-30 min.

\section{Data analysis and interpretation}

A special workstation using special software (Advantage Windows VS4; GE Healthcare, Wisconsin, USA) was used for the reconstruction of PET image data sets using the CT data for attenuation correction and image fusion to obtain co-registered multiplanar PET-CT images.

Two conjoint radiologists (5 years of experience in the PET/CT imaging) revised the CT, PET, and the fused $\mathrm{PET} / \mathrm{CT}$ images using visual assessment (considering the background activity as a standard reference for the same patient) and semi-quantitative assessment (by measuring the standardized uptake value (SUV) which was automatically calculated by special software).

The clinical and/or PSA level and/or radiological follow-up and/or the histopathological assessment for the patients served as a reference in the present study.

\section{Interpretation of the PET/CT findings}

The prostatic operative bed soft tissue masses or irregular soft tissue thickening were considered as positive for residual/recurrent neoplastic lesions if their tracer uptake was higher than the background activity.
Table 2 Treatment methods for the patients in the study with demonstration of their number (No.) and percent (\%)

\begin{tabular}{lll}
\hline Treatment method(s) & No. of patients & $\%$ of patients \\
\hline Operative intervention & 2 & $6.6 \%$ \\
Chemotherapy & 18 & $60 \%$ \\
Radiotherapy & 22 & $73.3 \%$ \\
Hormonal therapy & 15 & $50 \%$
\end{tabular}

N.B Most of patients recieved more than one method of treatment

If the patient did not have operation, the prostatic tracer uptake was considered as positive for residual/recurrent neoplastic lesions if its tracer uptake was higher than the background activity. A quantitative SUV cut-off value for prostatic cancer lesions does not exist [30].

Any lymph node with tracer activity more than the background activity was considered metastatic even if it was subcentemetric.

The pulmonary nodules that had a size of $5 \mathrm{~mm}$ or more were considered as positive for malignancy if their tracer uptake was exceeding the background activity; nevertheless, a metastatic disease could not be completely ruled out if the pulmonary nodules were less than $5 \mathrm{~mm}$ in their size.

If the bone marrow exhibited an obvious focal tracer avidity, we looked in CT to exclude benign bony lesions that may show increased 68Ga-PSMA uptake like Paget disease or healing fractures; otherwise, it was considered as positive for infiltration.

\section{Statistical methods}

Data management and analysis were performed using the Statistical Package for Social Sciences (SPSS) vs. 23. Numerical data were summarized using means, standard deviations, and ranges, as appropriate. Categorical data were summarized as numbers and percentages. The data

Table 3 Patterns of tumor recurrence/residual including lymph nodes and distant metastases by 68Ga-labelled PSMA-ligand PET/CT study with their number (No.) and percent (\%)

\begin{tabular}{lll}
\hline Patterns & No. of patients & \% of patients \\
\hline I) Operative bed/ prostatic residual or recurrence & 23 & $76.6 \%$ \\
II) Lymph nodes & 7 & $23.3 \%$ \\
a) Pelvi-abdominal & 5 & $16.6 \%$ \\
b) Mediastinal & 5 & $16.6 \%$ \\
c) Cervical & 0 & $0 \%$ \\
III) Distant metastasis & 11 & $36.6 \%$ \\
a) Liver & 0 & $0 \%$ \\
b) Lung & 3 & $10 \%$ \\
c) Bone & 10 & $33.3 \%$ \\
d) Brain & 0 & $0 \%$ \\
e) Others(adrenal) & 1 & $3.3 \%$ \\
\hline
\end{tabular}




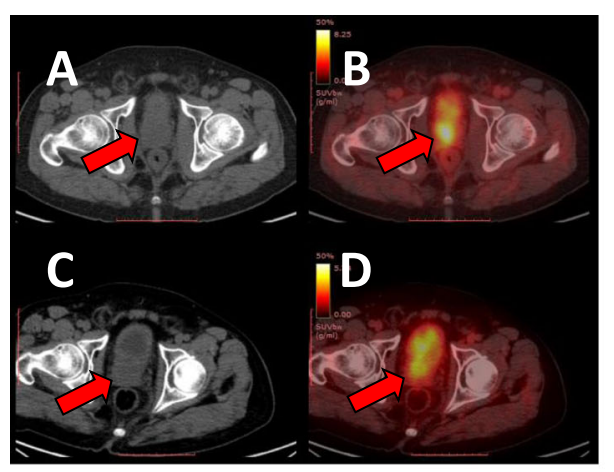

\section{Baseline \\ Follow up}

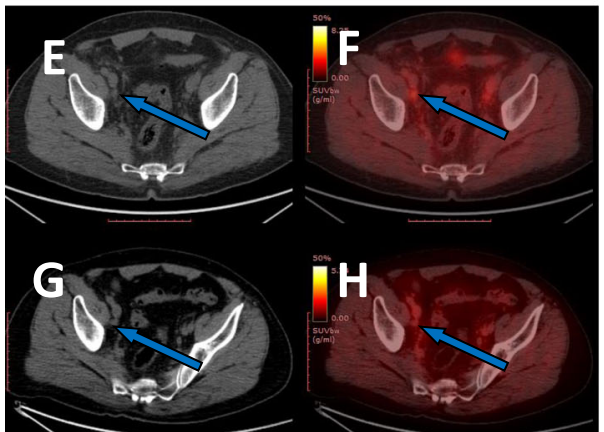

\section{Baseline}

\section{Follow up}

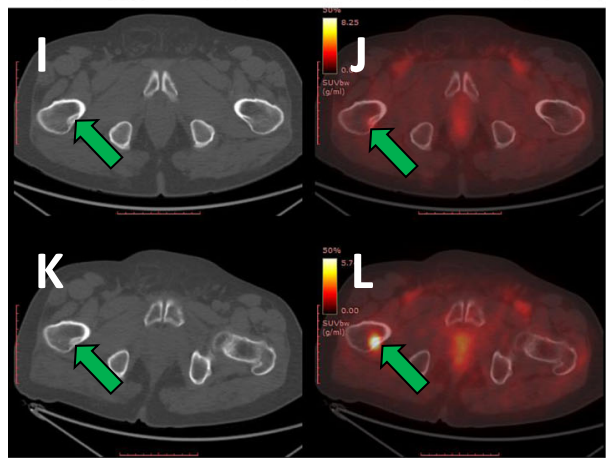

\section{Baseline}

\section{Follow up}

Fig. 1 Axial non-contrast CT and PET-CT images for a 62-year-old male patient with history of prostate cancer for which he had chemotherapy and radiotherapy. Images $\mathbf{a}$ and $\mathbf{b}$ (baseline study) showed focal prostatic 68Ga-PSMA activity with SUVmax $=9.2$ denoting residual neoplastic lesion that showed activity regression in images $\mathbf{c}$ and $\mathbf{d}$ (follow up study) showing SUVmax $=3.4$ (red arrows). Images $\mathbf{e}$ and $\mathbf{f}$ (baseline study) showed metastatic low-grade 68Ga-PSMA activity by subcentimetric right external iliac lymph node with SUVmax $=3.5$ and showed activity regression in images $\mathbf{g}$ and $\mathbf{h}$ (follow up study) with SUVmax $=1.7$ (blue arrows). Images $\mathbf{i}$ and $\mathbf{j}$ (baseline study) showed small right femoral neck sclerotic lesion with minimal 68Ga-PSMA activity that was considered insignificant, yet it showed activity progression in images $\mathbf{k}$ and $\mathbf{I}$ (follow up study) with SUVmax $=9.6$ (green arrows)

were collected, analyzed, and tabulated. The sensitivity, specificity, positive predictive value (PPV), negative predictive value (NPV), and the accuracy of the PET/CT in the diagnosis of recurrent prostatic cancer were calculated using the standard definitions [31].

\section{Results}

This study included 30 patients; age ranged from 53 to 80 years (mean 67.86) (Table 1 ).

All patients were treated by one or more of the following treatment methods: operative intervention, chemotherapy, radiotherapy, and hormonal therapy (Table 2).
Residual/recurrent malignancy was categorized as operative bed/prostatic recurrence/residual lesions, metastatic lymph nodes, and distant metastatic lesions (Table 3 and Figs. 1, 2, 3, 4, and 5).

Using the clinical and/or PSA level and/or radiological follow-up and/or the histopathological assessment for the patients as a reference, the 68Ga-labeled PSMA-ligand PET/CT (for the tumor recurrence/residual) had sensitivity, specificity, positive predictive value, negative predictive value, and an overall accuracy $(96.5 \%, 100 \%, 100 \%, 50 \%$, and 96.6\%) respectively (Tables 4 and 5). 


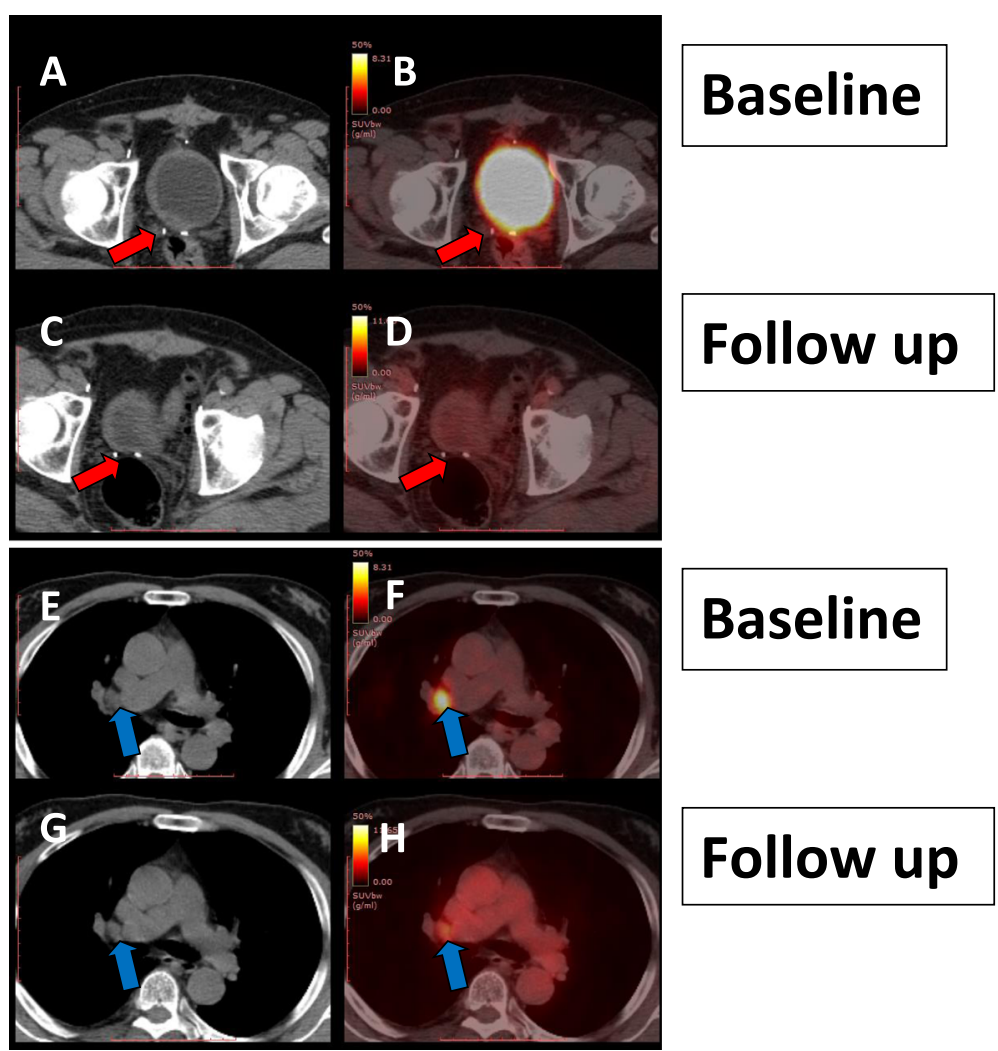

Fig. 2 Axial non-contrast CT and PET-CT images for a 72-year-old male patient with history of prostate cancer for which he had operation, chemotherapy, and hormonal therapy. Images $\mathbf{a}$ and $\mathbf{b}$ (baseline study) showed no 68Ga-PSMA activity at the operative bed with no significant changes in images $\mathbf{c}$ and $\mathbf{d}$ (follow up study) (red arrows). Images $\mathbf{e}$ and $\mathbf{f}$ (baseline study) showed 68Ga-PSMA activity by enlarged metastatic right hilar lymph node measuring $14 \mathrm{~mm}$ with SUVmax = 11.7 and showed activity regression in images $\mathbf{g}$ and $\mathbf{h}$ (follow up study) with SUVmax $=7.3$ (blue arrows)

\section{Discussion}

The highly promising method (68Ga-labeled PSMAligand PET/CT) proved to have a significant step forward in the diagnosis of recurrent prostatic cancer [20-22, 32]. This imaging modality is based on the observation that most adenocarcinomas of the prostate gland express PSMA to which 68Ga-labeled PSMAligand is able to bind with very high affinity [33, 34].

In our study, 68Ga-labeled PSMA-ligand PET/CT was positive for tumor recurrence (either operative bed, nodal, or distant metastases) in $28(96.5 \%)$ of patients and negative in 2 (3.5\%) of patients; however, AfsharOromieh et al. [30] who studied 319 patients with prostatic cancer and underwent treatment found that $68 \mathrm{Ga}-$ labeled PSMA-ligand PET/CT imaging showed a positive diagnosis in $264(82.8 \%)$ patients and a negative diagnosis in 55 (17.2\%) patients. Also, Matthias et al. [35] who studied 248 patients with prostatic cancer and underwent radical prostatectomy noticed that $68 \mathrm{Ga}$ labeled PSMA-ligand PET/CT imaging was negative in 26 (10.5\%) patients and positive in $222(89.5 \%)$ patients, while Mattiolli et al. [36] who studied 125 patients with prostatic cancer and underwent treatment showed that 68Galabeled PSMA-ligand PET/CT resulted in a positive diagnosis in $80(64 \%)$ patients and a negative diagnosis in 25 (36\%) patients (these differences in the results may be due to small sample size in the present study).

In the current work, 68Ga-labeled PSMA-ligand PET/ $\mathrm{CT}$ detected the operative bed/prostatic neoplastic residual or recurrence in $23(76.6 \%)$ patients, abdominopelvic nodal metastases in $5(16.6 \%)$ patients, mediastinal nodal metastases in $5(16.6 \%)$ patients, lung metastases in $3(10 \%)$ patients, bone metastases in $10(33.3 \%)$ patients, and adrenal metastases in $1(3.3 \%)$ patient denoting that pelvi-abdominal lymph node and bone metastases are the more frequent metastases in prostate cancer. These results agreed with Matthias et al. [35] who noticed that 68Ga-labeled PSMA-ligand PET/CT imaging detected operative bed local recurrence after radical prostatectomy in 87 (35.1\%) patients, abdominopelvic nodal metastases in 130 (52.4) patients, supradiaphragmatic nodal metastases in 13 (5.2\%) patients, bone metastases in 89 (35.9\%) patients, and liver/lung metastases in $13(5.2 \%)$ patients. Also, in a multicentric 

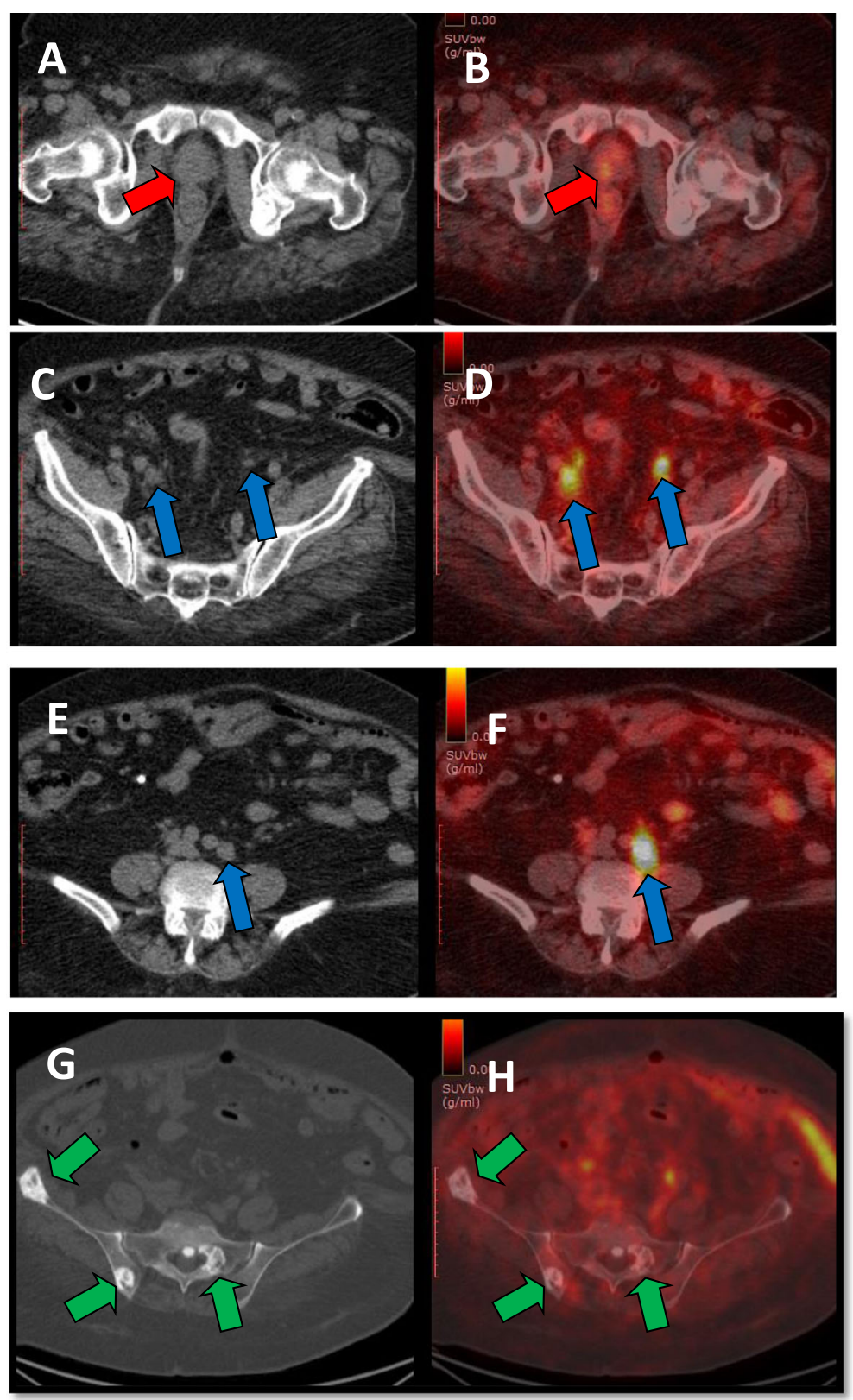

Fig. 3 Axial non-contrast CT and PET-CT images for a 79-year-old male patient with history of prostate cancer for which he had radiotherapy and hormonal therapy. Images $\mathbf{a}$ and $\mathbf{b}$ showed 68Ga-PSMA activity by small prostatic lesion with SUVmax $=2.5$ (red arrows) suggesting small residual neoplastic lesion. Images c-f showed 68Ga-PSMA activity by multiple small bilateral external and common iliac LNs; the most active shows SUVmax = 15.4 (blue arrows). The PET-CT revealed metastatic deposits in the iliac lymph nodes despite their subcentimetric size in CT. Images $\mathbf{g}$ and $\mathbf{h}$ showed multiple sclerotic osseous lesions involving the right iliac bone and sacrum with no 68Ga-PSMA activity (green arrows) denoting inactive metastatic lesions

study done by Mattiolli et al. [36], 68Ga-labeled PSMAligand PET/CT detected local recurrence in 38 (30.9\%) patients, loco-regional lymph node metastases in 55 (44.4\%) patients, extra-pelvic abdominal lymph node metastases in $15(12.5 \%)$ patients, thoracic lymph node metastases in $22(17.5 \%)$ patients, visceral metastases in 17 (13.6\%) patients, and bone metastases in 37 (29.6\%) patients, and these results are close to our study results.
In our study, our results demonstrated that $68 \mathrm{Ga}$ labeled PSMA-ligand PET/CT had sensitivity, specificity, PPV, NPV, and accuracy of $96 \%, 100 \%, 100 \%, 50 \%$, and $96.6 \%$ respectively. These results were close to the results reached by Afshar-Oromieh et al. [30] who studied 319 patients with prostatic cancer and underwent treatment and had 68Ga-labeled PSMA-ligand PET/CT examination and the PET-CT results were correlated 


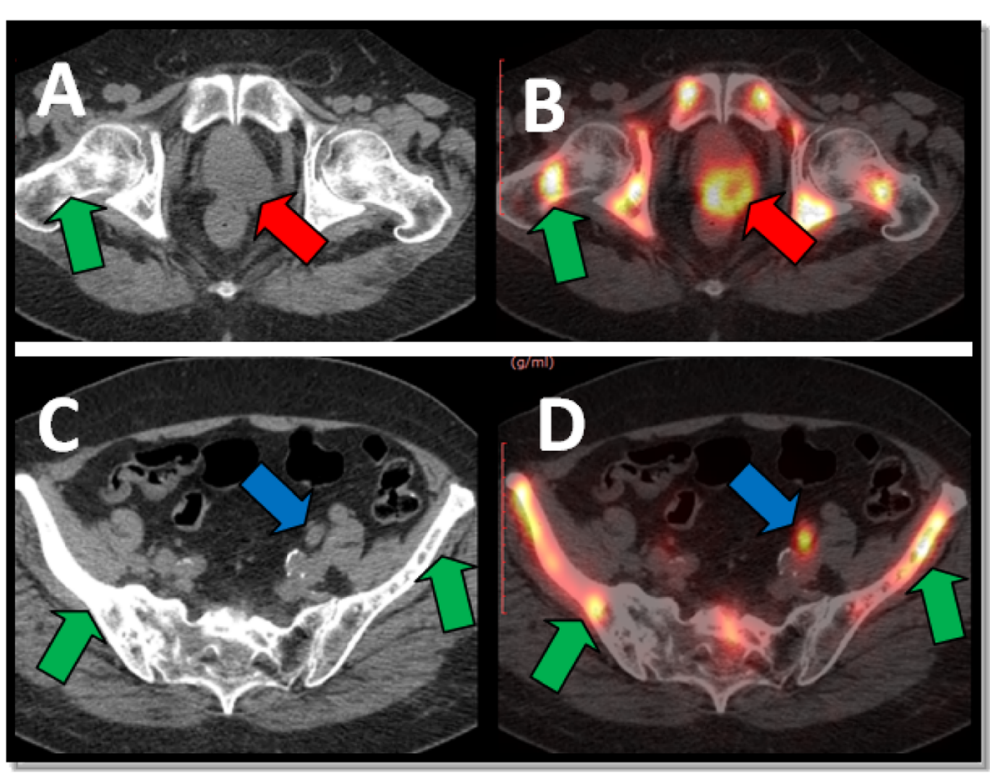

Fig. 4 Axial non-contrast CT and PET-CT images for a 60-year-old male patient with history of prostate cancer for which he had chemotherapy and radiotherapy. Images $\mathbf{a}$ and $\mathbf{b}$ showed prostatic lesion with 68Ga-PSMA activity with SUVmax $=17.5$ with posterior extra-prostatic extension and obliteration of the recto-prostatic space (red arrows), denoting residual neoplastic lesion. Images $\mathbf{c}$ and $\mathbf{d}$ showed 68Ga-PSMA activity by metastatic left external iliac lymph node with SUVmax = 10.6 (blue arrows). Images $\mathbf{a}-\mathbf{d}$ showed 68Ga-PSMA activity by metastatic sclerotic osseous lesions involving the pelvic bones (green arrows)

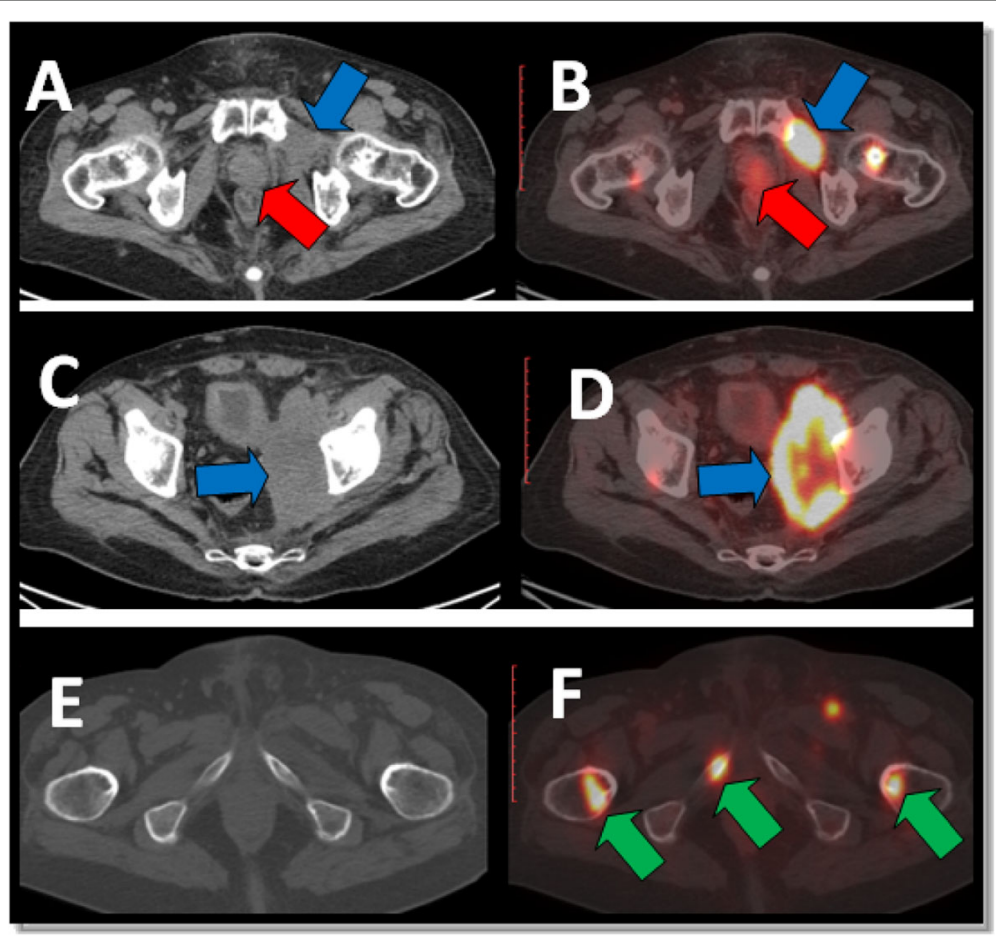

Fig. 5 Axial non-contrast CT and PET-CT images for a 61-year-old male patient with history of prostate cancer for which he had radiotherapy and hormonal therapy. Images $\mathbf{a}$ and $\mathbf{b}$ showed focal prostatic 68Ga-PSMA activity with SUVmax $=4.7$ (red arrows) denoting residual neoplastic lesion. Images a-d showed marked 68Ga-PSMA activity by metastatic left iliac nodal mass lesion encroaching on the left obturator and sacrosciatic foramina with SUVmax $=50.1$ (blue arrows). Images $\mathbf{e}$ and $\mathbf{f}$ showed marked 68Ga-PSMA activity by metastatic osseous lesions involving the right inferior pubic ramus and both proximal femora, the most active shows SUVmax $=45.4$ (green arrows) with no gross corresponding CT findings 
Table 4 Tumor recurrence/residual detected by 68Ga-labelled PSMA-ligand PET/CT in reference to the clinical and/or PSA level and/or radiological follow-up and/or the histopathological assessment for the patients, with the number (No.), percent (\%) of the patients

\begin{tabular}{|c|c|c|c|c|}
\hline $\begin{array}{l}\text { Tumoural residual/ } \\
\text { recurrence by PET/CT }\end{array}$ & $\begin{array}{l}\text { Positive by the } \\
\text { methods }(n=2\end{array}$ & ference & $\begin{array}{l}\text { Negative by th } \\
\text { reference meth } \\
(n=1)\end{array}$ & lods \\
\hline Positive $(n=28)$ & 28 patients (TP) & $96.5 \%$ & 0 patient (FP) & $0 \%$ \\
\hline Negative $(n=2)$ & 1 patient (FN) & $3.5 \%$ & 1 patient (TN) & $100 \%$ \\
\hline
\end{tabular}

$T N$ true negative, $T P$ true positive, $F N$ false negative, $F P$ false positive

with the histopathological examination in 42 patients and found patient-based sensitivity about $88.1 \%$ and lesionbased sensitivity, specificity, PPV, and NPV about 76.6\%, $100 \%, 100 \%$, and $91.4 \%$ in detecting recurrent disease in these patients. Also, Fitzpatrick et al. [37] did survey for 24 studies investigating the accuracy of 68Ga-labeled PSMAligand $\mathrm{PET} / \mathrm{CT}$ in detecting recurrent prostate cancer lesions and found that PET/CT was associated with sensitivity and specificity values of 33-93\% and > 99\% respectively and these results agreed with our study. Also, our results agreed with Fourquet et al. [38] who studied the impact of 68Ga-labeled PSMA-ligand PET/CT in restaging 30 patients with castration-resistant non-metastatic prostate cancer and found that the overall sensitivity and specificity of 68Ga-labeled PSMA-ligand PET/CT in detecting residual disease were $87 \%$ and $100 \%$ respectively.

This study had some limitations like the retrospective nature, small number of patients due to limited availability of the $68 \mathrm{Ga}$ PSMA radiotracer, and expensive cost of the scan. Further studies with larger sample of patients will give us more information about the role of this tracer in detecting recurrent prostate cancer. Also, we recommend comparative studies between 68Ga-labeled PSMA-ligand PET/CT and 99Tc bone scan for skeletal imaging in prostate cancer.

\section{Conclusion}

Our study showed 68Ga-PSMA PET/CT is a very helpful tool for detection of prostatic cancer recurrence and restaging which can help in performing treatment plans and improve the clinical outcome and survival of the patients.

Table 5 Accuracy measures for tumoural residual recurrence as detected by 68Ga-labelled PSMA-ligand PET/CT

\begin{tabular}{ll}
\hline Statistical parameter & Value \\
\hline Sensitivity & $96.5 \%$ \\
Specificity & $100 \%$ \\
Positive predictive value & $100 \%$ \\
Negative predictive value & $50 \%$ \\
Accuracy & $96.6 \%$ \\
\hline
\end{tabular}

\section{Abbreviations}

PET/CT: Positron emission tomography/computed tomography; 68Ga: 68 Gallium; PSMA: Prostatic-specific membrane antigen; PSA: Prostate-specific antigen; FDG: Fluoro-2-deoxy-D-glucose; SUV: Standardized uptake value

\section{Acknowledgements}

Not applicable.

\section{Authors' contributions}

All authors had actively contributed to the work. TAH contributed to the idea of study and data collection, obtained the radiological data in this study, shared in the design and coordination of the study, revised the obtained results, contributed to image revision, and did drafting the manuscript. MSE revised the obtained results and data and final editing. All authors read and approved the final manuscript.

\section{Funding}

No funding was received for this work from any organization.

\section{Availability of data and materials}

The datasets used and/or analyzed during the current study are available from the corresponding author on reasonable request.

\section{Ethics approval and consent to participate}

The protocol was reviewed and approved by the local ethics committee of the radiology department, Kasr Aliny hospital, Cairo University. The reference number was not applicable. All patients had given their written consents to participate in this work.

\section{Consent for publication}

All patients had given their written consent for publication of this work.

\section{Competing interests}

The authors declare that they have no competing interests.

\section{Author details}

${ }^{1}$ Radiology Department, Faculty of Medicine, Cairo University, Cairo, Egypt.

${ }^{2}$ Radiology Department, National Cancer Institute, Cairo University, Cairo, Egypt.

Received: 9 April 2020 Accepted: 16 July 2020

Published online: 23 July 2020

References

1. Siegel R, Ma J, Zou Z, Jemal A (2014) Cancer statistics. CA Cancer J Clin 64 9-29

2. Aus G, Abbou CC, Bolla M, Heidenreich A, Schmid HP, Van Poppel H et al (2005) EAU guidelines on prostate cancer. Eur Urol 48:546-551

3. Kosuri S, Akhtar NH, Smith M, Osborne JR, Tagawa ST (2012) Review of salvage therapy for biochemically recurrent prostate cancer: the role of imaging and rationale for systemic salvage targeted anti-prostate specific membrane antigen radioimmunotherapy. Adv Urol 2012:921674

4. Bott SRJ (2004) Management of recurrent disease after radical prostatectomy. Prostate Cancer Prostatic Dis 7:211-216

5. Beer AJ, Eiber M, Souvatzoglou M et al (2011) Radionuclide and hybrid imaging of recurrent prostate cancer. Lancet Oncol 12:181-191

6. Oyen RH, Van Poppel HP, Ameye FE et al (1994) Lymph node staging of localized prostatic carcinoma with CT and CT-guided fine-needle aspiration biopsy: prospective study of 285 patients. Radiology 190:315-322

7. Liu Y, Zuckier LS, Ghesani NV (2010) Dominant uptake of fatty acid over glucose by prostate cells: a potential new diagnostic and therapeutic approach. Anticancer Res 30(2):369-374

8. Reinicke K, Sotomayor P, Cisterna P, Delgado C, Nualart F, Godoy A (2012) Cellular distribution of Glut-1 and Glut-5 in benign and malignant human prostate tissue. J Cell Biochem 113(2):553-562

9. Castellucci P, Picchio M (2013) 11C-choline PET/CT and PSA kinetics. Eur J Nucl Med Mol Imaging 40(suppl 1):S36-\$40

10. Krause BJ, Souvatzoglou M, Tuncel M et al (2008) The detection rate of [11C] choline-PET/CT depends on the serum PSA-value in patients with biochemical recurrence of prostate cancer. Eur J Nucl Med Mol Imaging 35: $18-23$ 
11. Hillier SM, Maresca KP, Femia FJ, Marquis JC, Foss CA, Nguyen N et al (2009) Preclinical evaluation of novel glutamate-urea-lysine analogues that target prostate-specific membrane antigen as molecular imaging pharmaceuticals for prostate cancer. Cancer Res 69:6932-6940

12. Eder M, Schäfer M, Bauder-Wüst U, Hull WE, Wängler C, Mier W et al (2012) 68Ga-complex lipophilicity and the targeting property of a urea based PSMA inhibitor for PET imaging. Bioconjug Chem 23:688-697

13. Schäfer M, Bauder-Wüst U, Leotta K, Zoller F, Mier W, Haberkorn U et al (2012) A dimerized urea-based inhibitor of the prostate-specific membrane antigen for 68Ga-PET imaging of prostate cancer. EJNMMI Res 2:23

14. Bander NH (2006) Technology insight: monoclonal antibody imaging of prostate cancer. Nat Clin Pract Urol 3:216-225

15. Liu H, Moy P, Kim S, Xia Y, Rajasekaran A, Navarro V et al (1997) Monoclonal antibodies to the extracellular domain of prostate specific membrane antigen also react with tumor vascular endothelium. Cancer Res 57:3629-3634

16. Sweat SD, Pacelli A, Murphy GP, Bostwick DG (1998) Prostate-specific membrane antigen expression is greatest in prostate adenocarcinoma and lymph node metastases. Urology 52:637-640

17. Mannweiler S, Amersdorfer P, Trajanoski S, Terrett JA, King D, Mehes G (2009) Heterogeneity of prostate-specific membrane antigen (PSMA) expression in prostate carcinoma with distant metastasis. Pathol Oncol Res 15:167-172

18. Chen Y, Pullambhatla M, Foss CA, Byun Y, Nimmagadda S, Senthamizhchelvan S et al (2011) 2-(3-\{1-Carboxy-5-[(6-[18F]fluoro-pyridine3-carbonyl)-amino]-pentyl\}-ureido)-pen tanedioic acid,[18F]DCFPyL, a PSMAbased PET imaging agent for prostate cancer. Clin Cancer Res 17:7645-7653

19. Eder M, Eisenhut M, Babich J, Haberkorn U (2013) PSMA as a target for radiolabelled small molecules. Eur J Nucl Med Mol Imaging 40:819-823

20. Afshar-Oromieh A, Haberkorn U, Schlemmer HP, Fenchel M, Eder M, Eisenhut $M$ et al (2014) Comparison of PET/CT and PET/MRI hybrid systems using a (68)Ga-labelled PSMA ligand for the diagnosis of recurrent prostate cancer: initial experience. Eur J Nucl Med Mol Imaging 41:887-897

21. Afshar-Oromieh A, Malcher A, Eder M, Eisenhut M, Linhart HG, Hadaschik BA et al (2013) PET imaging with a [68Ga]gallium-labelled PSMA ligand for the diagnosis of prostate cancer: biodistribution in humans and first evaluation of tumour lesions. Eur J Nucl Med Mol Imaging 40:486-495

22. Afshar-OromiehA ZCM, MalcherA EM, Eisenhut M, Linhart HG et al (2014) Comparison of PET imaging with a (68)Ga -labelled PSMA ligand and (18)Fcholine-based PET/CT for the diagnosis of recurrent prostate cancer. Eur J Nucl Med Mol Imaging 41:11-20

23. Zechmann CM, Afshar-Oromieh A, Armor T, Stubbs JB, Mier W, Hadaschik B et al (2014) Radiation dosimetry and first therapy results with a (124)I/(131)|labeled small molecule (MIP-1095) targeting PSMA for prostate cancer therapy. Eur J Nucl Med Mol Imaging 41:1280-1292

24. Lapi SE, Wahnishe H, Pham D, Wu LY, Nedrow-Byers JR, Liu T et al (2009) Assessment of an 18F-labeled phosphoramidate peptidomimetic as a new prostate-specific membrane antigen targeted imaging agent for prostate cancer. J Nucl Med 50:2042-2048

25. Holland JP, Divilov V, Bander NH, Smith-Jones PM, Larson SM, Lewis JS (2010) 89Zr-DFO-J591 for immunoPET of prostate-specific membrane antigen expression in vivo. J Nucl Med 51:1293-1300

26. Demirci E, Sahin OE, Ocak M, Akovali B, Nematyazar J, Kabasakal L (2016) Normal distribution pattern and physiological variants of 68Ga-PSMA-11 PET/CT imaging. Nucl Med Commun 37(11):1169-1179

27. Wallitt KL, Khan SR, Dubash S, Tam HH, Khan S, Barwick TD (2017) Clinical PET Imaging in Prostate Cancer. Radiographics 37:1512-1536

28. Krohn T, Verburg FA, Pufe T et al (2015) [68Ga] PSMA-HBED uptake mimicking lymph node metastasis in coeliac ganglia: an important pitfall in clinical practice. Eur J Nucl Med Mol Imaging 42(2):210-214

29. Beheshti M, Rezaee A, Langsteger W (2017) 68Ga-PSMA-HBED uptake on cervicothoracic (stellate) ganglia, a common pitfall on PET/CT. Clin Nucl Med 42(3):195-196

30. Afshar-Oromieh A, Avtzi E, Giesel FL, Holland-Letz T, Linhart HG, Eder M, Eisenhut M, Boxler S, Hadaschik BA, Kratochwil C, Weichert W, Kopka K, Debus J, Haberkorn U (2015) The diagnostic value of PET/CT imaging with the 68Ga-labelled PSMA ligand HBED-CC in the diagnosis of recurrent prostate cancer. Eur J Nucl Med Mol Imaging 42:197-209

31. Galen RS (1980) Predictive values and efficiency of laboratory testing. Pediat J Clin North Am 27:861-869

32. Roethke MC, Kuru TH, Afshar-Oromieh A, Schlemmer HP, Hadaschik BA, Fenchel M (2013) Hybrid positron emission tomography-magnetic resonance imaging with gallium 68 prostate-specific membrane antigen tracer: a next step for imaging of recurrent prostate cancer-preliminary results. Eur Urol 64:862-864

33. Ross JS, Sheehan CE, Fisher HA, Kaufman RP Jr, Kaur P, Gray K et al (2003) Correlation of primary tumor prostate-specific membrane antigen expression with disease recurrence in prostate cancer. Clin Cancer Res 9: $6357-6362$

34. Silver DA, Pellicer I, Fair WR, Heston WD, Cordon-Cardo C (1997) Prostatespecific membrane antigen expression in normal and malignant human tissues. Clin Cancer Res 3:81-85

35. Eiber M, Maurer T, Souvatzoglou M, Beer AJ, Ruffani A, Haller B, Graner F-P, Kübler H, Haberhorn U, Eisenhut M, Hans-JürgenWester, Gschwend JE, Schwaiger M (2015) Evaluation of hybrid 68Ga-PSMA ligand PET/CT in 248 patients with biochemical recurrence after radical prostatectomy. J Nucl Med 56:668-674

36. Mattiolli AB, Santos A, Vicente A, Queiroz M, Bastos D, Herchenhorn D, Srougi M, Peixoto FA, Morikawa L, da Silva JLF, Etchebehere E (2018) Impact of 68GA-PSMA PET /CT on treatment of patients with recurrent / metastatic high risk prostate cancer - a multicenter study. Int Braz J Urol 44:892-899

37. Fitzpatrick L, Lynch O, Marignol L (2017) 68Ga-PSMA-PET/CT has a role in detecting prostate cancer lesions in patients with recurrent disease. Anticancer Res 37:2753-2760

38. Aloÿse Fourquet, Cyrielle Aveline, Olivier Cussenot, Gilles Créhange, Françoise Montravers,Jean-Noël Talbot, Mathieu Gauthé (2020) 68Ga-PSMA$11 \mathrm{PET} / \mathrm{CT}$ in restaging castration-resistant nonmetastatic prostate cancer: detection rate, impact on patients' disease management and adequacy of impact 10:2104

\section{Publisher's Note}

Springer Nature remains neutral with regard to jurisdictional claims in published maps and institutional affiliations.

\section{Submit your manuscript to a SpringerOpen ${ }^{\circ}$ journal and benefit from:}

- Convenient online submission

- Rigorous peer review

- Open access: articles freely available online

High visibility within the field

- Retaining the copyright to your article

Submit your next manuscript at $\boldsymbol{\nabla}$ springeropen.com 